\title{
UCLA
}

Paroles gelées

Title

Rhétorique verbale et visuelle dans les Salons de Diderot

Permalink

https://escholarship.org/uc/item/41c7h4p0

Journal

Paroles gelées, 21(1)

ISSN

1094-7264

Author

Hakim, Zeina

Publication Date

2004

DOI

10.5070/PG7211003156

Peer reviewed 


\section{RHÉTORIQUE VERBALE ET VISUELLE DANS LES $S$ ALONS DE DIDEROT}

Zeina Hakim is a doctoral candidate in the Department of French. Columbia University.

Le grand homme n'est pas celui qui fait vrai, c'est celui qui sait le mieux concilier le mensonge avec la vérité.

DIDEROT, Salon de 1767.

Ce fut la rédaction des Salons et les observations et réflexions qu'elle rendit nécessaires qui amenèrent Diderot à ne plus seulement considérer l'art comme une reproduction exacte de la nature, mais bien comme une transformation personnelle de certains éléments soigneusement choisis dans le réel. La lecture des Salons ne laisse pas de doute à ce sujet. En effet, Diderot ne se contente pas d'y être un technicien qui décrit et commente une composition d'un air détaché. Il ne se contente pas non plus de refaire les tableaux par des digressions expressives, moins encore d'en donner un simple compte-rendu. Il s'en sert tout simplement pour créer un monde à part, oscillant entre réalité et fiction, dans lequel son imagination peut errer à sa guise en créant d'autres réalités.

Par la substitution expressive, par le dialogue imaginaire, par son identification avec tel ou tel personnage ou telle ou telle situation représentée sur la toile, Diderot essaye, dans les Salons, de forger l'unité de l'expression picturale et de l'expression littéraire. Il trouve possible de se promener dans les compositions d'un peintre, d'imaginer qu'il vit et se meut dans le paysage que celui-ci a mis sur la toile: il se trouve fictivement transporté dans le tableau. Diderot met en scène une mystification éblouissante, qui doit son pouvoir envoûtant à la démystification que son lecteur attend, et que Diderot retarde savamment. L'effet de réel est avivé par la conscience d'illusion. D’où la possibilité inverse de percevoir la réalité extérieure comme une illusion esthétique. Nous voyons Diderot se dresser en accusateur du peintre et même apostropher les personnages d'un tableau. Par ailleurs, Diderot, dans ses Salons, met en scène la fiction d'une présence physique d'un spectateur dans le tableau. Il s”identifie avec l'action qu'il décrit, soit comme participant, soit comme spectateur unique et insoupçonné: dans les deux cas, il désire s'intégrer à la composition. C’est précisément ce procédé assurant la convergence du texte et de la peinture que je souhaite analyser ici.

Par ailleurs, Diderot a constamment recours à la conversation pour parler de l'art. Le dialogue qu'il instaure avec le peintre du tableau, ses personnages ou un interlocuteur fictif semble lui permettre un déplacement du plan réel au plan de fiction. Or, comment un tel déplacement est-il rendu possible par Diderot? Quelles stratégies narratives, rhétoriques ou stylistiques met-il en scène pour décrire l'abolition des frontières entre réalité et fiction? Enfin, quelles sont les visées profondes d'une telle abolition - si celle-ci s'avère possible ?

Pour répondre à ces interrogations, $\mathrm{j}$ 'analyserai au cours de cet article les traits qui contribuent, dans les Salons, à fragiliser les limites entre le réel et le fictif. Il me sera alors possible de voir en quoi la mise en scène de Diderot relève d'une rhétorique à la fois verbale et visuelle. 
Les adresses faites aux destinataires des Salons Diderot et Grimm

Le destinataire principal des Salons est l'ami Grimm. Mais. outre le Grimm aux sollicitations duquel Diderot répond pour les lecteurs de la Correspondance littéraire, un autre Grimm est déjà présent dans le texte des Salons: celui que Diderot fait parler fictivement, celui dont il joue librement, comme d'un faire-valoir, parmi d'autres interlocuteurs occasionnellement inventés. Diderot a eu recours à un certain nombre d'artifices susceptibles de produire l'illusion de présence: l'un d'eux, qu'il utilise de façon constante. est le recours au dialogue supposé et à l'apostrophe. Ainsi, le dialogue avec Grimm reste un élément constitutif du style: " $C$ "est une belle chose, mon ami, que les voyages... " (Diderot $3: 221$ ), "O mon cher Grimm, la belle occasion que cet artiste a manquée... " (Diderot 3: 289).

Par ailleurs, Grimm se trouve crédité de tous les progrès réalisés par Diderot. Il a part à l'œuvre accomplie, il y a même droit de regard. Diderot lui concède le pouvoir de le corriger, de le remanier, de le censurer: "Coupez, taillez, tranchez, rognez et ne laissez de tout cela que ce qui vous duira" (Diderot 3: 113). Diderot s'adresse à lui pour introduire le texte et pour y établir le rapport personnel si nécessaire pour la libre expression des jugements qui vont suivre. De même. Grimm ne se prive pas d'insérer ses commentaires, ses restrictions, ses éclaircissements. Il tient, dans le duo, une partie d'accompagnement: il garde la tête froide: il est perspicace, souvent péremptoire et sarcastique ${ }^{1}$. Il se permet de commenter le travail de Diderot grâce à une formule qui prévaudra dans la présentation des Salons: "C'est lui qui va prendre la plume. Je marquerai d'une étoile les notes que je me permettrai d'ajouter par-ci par-là à son travail» (Diderot 1: 118). Ces notes insérées dans le texte sont importantes non seulement par leur étendue, mais aussi parce qu'elle développent - ou réfutent - les jugements de Diderot et transforment çà et là son monologue en dialogue. Ainsi s'instaure un double regard sur les œuvres exposées. Le Grimm " réel » n'a donc pas uniquement une fonction de censeur pouvant supprimer ou atténuer certains passages mais il joint à celle-ci la fonction de commentateur.

Le commentaire du tableau intitulé Le grand-prêtre Corésus s'immole pour sauver Callirho $\dot{e}^{2}$ et présenté par Fragonard au Salon de 1765. est, à cet égard, particulièrement intéressant puisqu'il est construit comme un vaste dialogue où le personnage de Grimm joue un rôle très actif. En effet, Diderot, dirigeant son réve de manière à le faire aboutir à la scène du sacrifice telle que l'a peinte Fragonard, a besoin qu'un autre personnage intervienne, pour attester que la fin du rêve, tel que Diderot le raconte, et le tableau de Fragonard, coïncident parfaitement. 11 a besoin d'un partenaire fictif, qui puisse effectuer la comparaison. Et comme c'est à Grimm qu'il envoie son Salon, il lui suffira de doubler le Grimm réel d'un Grimm imaginaire, qu'il fera parler à sa guise. Ce Grimm second, interlocuteur imaginaire, accepte sans discussion l'histoire du rêve, et s'émerveillera d'en trouver la conclusion si semblable au tableau réel qu il a vu, qu'il a jugé, et dont le souvenir lui est resté dans tous ses détails. Le Grimm que fait parler Diderot commente ainsi la fin de la description de celui-ci:

Le temple que vous venez de décrire est exactement le lieu de la scène du tableau de Fragonard. [...] Vos personnages, ce sont là tous les 
Zeina Hakim

personnages du tableau de Fragonard, et ils se sont trouvés dans votre rêve, placés tout juste comme sur sa toile. [...] Voilà le tableau de Fragonard, le voilà dans tout son effet. C'est le même temple, la même ordonnance, les mêmes personnages, la même action, les mêmes caractères, le même intérêt général, les mêmes qualités, les mêmes défauts. (Diderot 2 : 194-195)

Le compte-rendu de Corésus et Callirhoé est donc présenté sous la forme d'un dialogue imaginaire entre Diderot et Grimm. Bien que ce soit Grimm qui sert de repoussoir aux élans de Diderot, ce dernier n’hésite pas à donner à son ami Grimm un rôle dans la distribution des éloges : "Quoique les juges en disent, croyez que vous avez fait un beau rêve, et Fragonard un beau tableau. [...] Comptez même que votre rêve est plus beau que son tableau " (Diderot $2: 198$ ).

\section{Diderot et ses destinataires}

Cependant, si les Salons forment, dans leur ensemble, un dialogue continuel, Diderot ne se limite pas à faire parler son ami Grimm. Les voix qu'il met en scène sont diverses: apostrophes éloquentes ou touchantes (chez Greuze), bruits de torrents (chez Loutherbourg), voix des oracles ou même (dans le Baptêne du Christ que Diderot récrit à la place de Brenet et de Lépicié) voix de Dieu criant du haut des cieux : "Celui-ci est mon fils bien-aimé !» (Diderot 2: 164). Ces bruits de voix, qui avaient toujours existé autour de la peinture, voici qu'avec Diderot ils deviennent pleinement audibles. En les orchestrant librement, dans leur polyphonie. Diderot annexe la critique d'art à la littérature. On conçoit dès lors la nécessité qu'il y a à prêter toute son attention à la polyphonie des Salons, composés de nombreuses voix attribuables, tantôt à des interlocuteurs implicites et présupposés, tantôt à des partenaires simulés par le critique d'art, metteur en scène de figures ennemies ou amies. ${ }^{3}$.

Chez Diderot, l'apostrophe à la deuxième personne du pluriel occupe une place importante, non seulement par la présence réelle ou fictive d’un destinataire, mais par l'habitude qu'il a d'apostropher les personnages dont il parle. Cette orientation stylistique s'applique assez souvent aux peintres, surtout quand Diderot n'en pense pas beaucoup de bien : "Monsieur Baudouin, faites-moi le plaisir de me dire en quel lieu du monde cette scène s'est passée» ((Diderot 3: 197); "Monsieur Fragonard, cela est diablement fade. Belle omelette, bien douillette, bien jaune et point brûlée" (Diderot $3: 280$ ); le plus mal loti étant sans doute le peintre Jean-Baptiste Pierre, premier peintre de M. le duc d'Orléans : "Sachez, Monsieur Pierre, qu'il ne faut pas copier ou copier mieux ; et de quelque manière qu'on fasse, il ne faut pas médire de ses modèles" (Diderot 1: 113). Mieux encore : quand le commentateur est pressé d'en finir. il s’adresse directement aux personnages du tableau. C'est ainsi qu'il apostrophe la trop jolic Madeleine peinte par Carle Van Loo: "Belle sainte, venez: entrons dans cette grotte, et là nous nous rappellerons peut-être quelques moments de votre première vie» (Diderot $1: 110$ ).

Mais le destinataire auquel s'adresse avant tout Diderot est son lecteur. Il souhaite amener celui-ci à voir avec ses yeux, à éprouver ses impressions; I'amener à entrer véritablement en communion avec lui. Ce qui n’est pas sans poser de sérieux 
problèmes d'écriture. Comment faire du texte l'équivalent de l'image ? Comment susciter chez l'autre les mêmes sentiments ? Comment èveiller en lui les mêmes réflexions? Faire de son lecteur un véritable spectateur du Salon devient la préoccupation essentielle. Pour cela. Diderot fait continuellement appel à son imagination. Il l'invite à se porter avec lui sur les lieux mêmes de l'exposition. à se placer devant la toile et à se laisser pénétrer par la magie des formes et des couleurs, à contempler, à écouter, à sentir, à rêver.

De plus, à plusieurs reprises dans ses Salons, Diderot cajole, réprimande, surprend et se moque tour à tour de son lecteur, lui donnant l'impression qu'il est de connivence avec lui dans une aventure toute personnelle. A d'autres moments, Diderot prend soin de décrire l'œuvre picturale tout en dialoguant avec lui-même pour anticiper les objections de son lecteur. D'un seul coup, la description est rendue vivante, doublement expressive:

Derrière Hèlène et Pâris, d'autres femmes, les yeux fixés sur Hector... Aurez-vous bientôt fini? Dites-vous? Attendez, attendez; vous n'y êtes pas. Sur un plan plus élevé, tout à fait sur la gauche, Vénus et son fils, apparemment sur un autel. M'avez-vous suivi? Cela s'est-il arrangé dans votre tête ?. (Diderot $2: 109$ )

Diderot semble rêver d'un texte qui se passe de toute description détaillée, d'un langage capable de susciter dans l'imagination du lecteur l'image même du tableau contemplé, d'une manière immédiate, sans passer par l'entremise des mots. Ou plutôt d'une description réduite à une simple esquisse capable de déclencher la fantaisie du lecteur et de conduire ce dernier à recréer le tableau.

L'impuissance des mots à rendre le spectacle et la poésie d'un grand tableau de Casanova (Une marche d'armée) le fait rêver de cette formule:

Comment vous donnerai-je une idée vraie de ce vieux château $[\ldots]$ ? Comment ferai-je descendre le torrent des montagnes, en précipiterai-je les eaux sous ce pont, et les répandrai-je tout autour du site élevé sur lequel la masse de pierre est construite ? Comment vous tracerai-je la marche de cette armée ? Mais peut-être qu'en désespérant de réaliser dans votre imagination tant d'objets animés, inanimés, ils le sont; et je l'ai bien fait. Si cela est, Dieu soit loué. (Diderot 2 : 132-133)

Mais il sait très bien que ce n'est qu'un rêve, et que cette communication où son lecteur et lui se retrouveraient côte à côte dans l'espace imaginaire n'est qu'une belle chimère. Aussi ne se tient-il pas quitte de sa tâche et, revenant à la réalité. il laisse de côté les élans poétiques et entreprend méthodiquement et froidement la description de la toile de Casanova, qu'il définit à la fin comme un "beau poème, bien conçu, bien conduit et mal écrit " (Diderot $2: 134$ ).

Ainsi, l'expérience de rédacteur des Salons constitue pour Diderot le laboratoire où il essaie de nouvelles techniques, telles que le goût du jeu et de la mystification. la confusion des plans qui le font passer de la réalité à la fiction et de la fiction à la réalité sans solution de continuité, le goût de la digression qui transforme ses récits en une conversation à bâtons rompus où passent les idées les plus disparates et en même temps 
les plus vraies. Ce sont là autant de procédés que, plus tard, il mettra à l'œuvre dans ses récits de fiction.

\section{La question de la pénétration fictive du narrateur dans le récit Une promenade picturale}

Mais, après avoir tenté de créer une communication directe avec son lecteur, Diderot va aller encore plus loin dans sa mystification en utilisant un nouveau procédé : l'entrée du lecteur dans le tableau. C'est le moment fatidique où la peinture, cessant d'être un objet, acquiert les qualités d'un sujet agissant et sentant. Cet événement s'est produit en 1767 quand Diderot s'est trouvé en face des Sites de Vernet, au nombre de six d'après son commentaire, qu'il a complété par un "septième tableau " dont il est difficile de dire s'il est de son invention ou non. A la vue de ces Sites, il transforme les tableaux en paysages réels. Dès la première phrase, la distance est en effet abolie. L'obstacle de la toile disparait: "J'avais écrit le nom de cet artiste en haut de ma page, lorsque je stis parti pour une campagne voisine de la mer et renommée pour la beauté de ses sites" (Diderot 3: 129). C'est une formule ingénieuse pour inviter son lecteur à se transporter par l'imagination devant le spectacle de la nature et à partager l'extase que lui-même a connue en admirant ces peintures dans le Salon. Au lieu de décrire les paysages que représentent les peintures exposées, il simule une promenade avec le précepteur des enfants du baron et ses deux élèves.

Au gré de leur marche, son accompagnateur et lui découvrent les paysages exposés par Vernet. Descriptions et entretiens sont à leur tour scandés par des rappels continuels du cadre réel dans lequel ils sont censés s insérer: le séjour au château; les haltes dans les promenades ou encore les jeux des enfants. Mais le plus intéressant est qu'après avoir fait une mise en scène impeccable, dans laquelle tout contribue à créer l'illusion de la réalité de la promenade et des paysages contemplés. Diderot abat ses cartes et dévoile son jeu à son ami Grimm : «Mon secret m’a échappé et il n'est plus temps de recourir après. Entrainé par le charme du Clair de Lune de Vernet, j’ai oublié que je vous avais fait un conte) (Diderot $3: 158$ ).

Pour présenter aux yeux de son lecteur les peintures de Vernet de la manière la plus vraie, Diderot a donc choisi de passer par le mensonge d'un récit de fiction. Ces promenades imaginaires lui permettent de faire un récit plus authentique que s'il était resté au Salon à décrire les tableaux. Car mieux que tout autre langage, elles traduisent sa véritable expérience de spectateur, les sentiments et les états d'âme que ces toiles ont éveillés en lui. Le conte de Vernet n'est cependant pas conçu pour tromper le lecteur. Aussi Diderot finit-il par montrer son jeu. Car au fond c'est d'un jeu qu'il s'agit, d'un jeu dans lequel le lecteur, à tout moment, est invité à participer. Dès les premières lignes, le récit est parsemé de petites phrases allusives qui sont là pour lui «donner l'éveil ", pour employer l'expression de Rameau. Ce sont des indices qui, dans l'esprit du narrateur, devraient mettre le lecteur averti sur la piste.

Cette anecdote renferme une des clés de l'écriture narrative de Diderot et de sa manière de concevoir la communication avec son lecteur. Dans le passage signalé, les propos échangés entre les promeneurs et l'admiration suscitée par les paysages se confondent avec l'émotion éprouvée au Salon devant les peintures de Vernet. Les deux expériences se superposent, s'amalgament et s'identifient. Dans le creuset de 
l'imagination. Diderot transforme le vécu en un récit fictif. Il a suffi d'un changement de décor pour que la réalité devienne fiction.

Cependant, ce procédé de pénétration fictive dans le récit n'est pas le seul moyen qu'utilise Diderot pour fragiliser la frontière entre fiction et réalité : ce qui accroît encore le plaisir du lecteur / spectateur, c'est le jeu de la chair frôlée par les draperies et les cheveux. c'est la chair touchée par une main. Ainsi, dans la Madeleine de Lagrenée: "Elle a les bras croisés sur sa poitrine. Ses longs cheveux viennent en serpentant dérober sa gorge. Comme dans sa douleur, ses bras se serrent sur sa poitrine et ses mains contre ses bras, l'extrémité de ses doigts s'enfonce légèrement dans sa chair " (Diderot $2: 95$ 96). Diderot semble, de plus, désirer s 'intégrer à la composition qu'il décrit: " $\mathrm{O}$ la belle main ! La belle main ! Le beau bras ! Voyez la vérité des détails de ces doigts : et ces fossettes et cette mollesse, et cette teinte de rougeur dont la pression de la tête a coloré le bout de ces doigts délicats, et le charme de tout cela. On s'approcherait de cette main pour la baiser, si on ne respectait cette enfant" (Diderot 2 : 145).

Ce qui ravit Diderot, en 1763, dans Chardin, c'est que la surface de la toile se laisse franchir dans les deux sens, par les objets représentés et par le spectateur : " C"est la nature même; les objets sont hors de la toile et d'une vérité à tromper les yeux. Pour regarder les tableaux des autres, il semble que $\mathrm{j}$ 'ai besoin de me faire des yeux : pour voir ceux de Chardin. je n’ai qu’à garder ceux que la nature $m$ 'a donnés et $m$ ’en bien servir " (Diderot $1: 222$ ). L'imitation parfaite fait du tableau le double tautologique de la réalité: l'objet n'est plus captif de la figuration, il devient disponible pour la main. pour l'usage pratique :

C'est que ce vase de porcelaine est de la porcelaine : c'est que ces olives sont réellement séparées de l'œil par l'eau dans laquelle elles nagent ; $c^{\prime}$ 'est qu'il n'y a qu'à prendre ces biscuits et les manger, cette bigarade, l'ouvrir et la presser, ces fruits et les peler, ce pâté et y mettre le couteau. (Diderot $1: 222$ )

Chardin est donc, pour Diderot, un grand magicien ; son " faire » est souverain, et le spectateur transporté par ses toiles n'est plus capable d’établir la différence entre l'olive réelle et l'olive représentée, la pêche du tableau et celle de la nature: "C"est toujours la nature et la vérité ; vous prendriez les bouteilles par le goulot, si vous aviez soif, les pêches et les raisins éveillent l'appétit et appellent la main » (Diderot 1:66).

A un degré plus élevé se situe la participation affective, étayée par la précision du compte rendu technique. Devant l'Accordée de Village, Diderot croit revoir le Père de Famille, il décrit la scène, il anime les personnages, il les fait parler, avec toutes les facilités que cela comporte: "Les deux servantes, debout, au fond de la chambre. nonchalamment penchées l'une contre l'autre, semblent dire, d'attitude et de visage : Quand est-ce que notre tour viendra? . Le père, quant à lui, les bras étendus vers son gendre, lui parle avec une effusion de cœur qui enchante et il semble lui dire: Jeannette est douce et sage, elle fera ton bonheur, songe à faire le sien...ou quelque autre chose sur l'importance des devoirs du mariage. Ce qu'il dit est sûrement touchant et honnête " (Diderot $1: 142$ ). Ce procédé poétique, qui consiste à entrer dans le tableau et à transformer le commentaire pictural en méditation, est particulier à Diderot: « Ah ! mon 
ami, que la nature est belle dans ce petit canton! Arrêtons-nous y ; la chaleur du jour commence à s'y faire sentir ! " (Diderot $1: 226)$.

Là où le tableau fait un appel très direct aux sens, Diderot "participe" également facilement à la composition. La "participation" de Diderot est parfois évidente :

Les détails, surtout au vieillard, sont admirables. Mais ce n’est pas là le tableau que $j$ 'ai dans l'imagination. Je ne veux pas que ce malheureux vieillard, ni cette fenme charitable, soupçonnent qu'on les observe ; ce soupçon arrête l'action et détruit le sujet. [...] Je ne veux pas que ce soit une jeune femme : il me faut une femmc au moins de trente ans, d'un caractère grand, sévère et honnête, que son expression soit celle de la tendresse et de la pitié. En un mot, je veux que cette scène soit traitée du plus grand style, et que d'un trait d'humanité pathétique et rare, on en $\mathrm{m}^{\circ}$ en fasse pas une petite chose. (Diderot 2:95)

On ne peut dès lors guère douter que Diderot s'identifie avec l'action qu'il décrit, soit comme participant, soit comme spectateur: dans les deux cas, il désire s`intégrer à la composition. Diderot rêve déjà ici à la toute-puissance du narrateur, capable de donner à voir des scènes imaginaires et de susciter de vrais sentiments au gré de sa fantaisie. Et déjà, au même moment, il s'amuse également à démystifier ce pouvoir du romancier. Le germe de l'écriture narrative de Jacques le fataliste se trouve à plusieurs endroits dans les Salons, comme c'est le cas par exemple dans la description des Sites de Vernet auquel je faisais référence un peu plus haut :

Je vous raconte simplement la chose. Dans un moment plus poétique j'aurais déchaîné les vents, soulevé les flots, montré la petite nacelle tantôt voisine des nues, tantôt précipitée au fond des abîmes: vous auriez frémi pour l'instituteur, ses jcunes éleves, et le vieux philosophe votre ami. J'aurais porté de la terrasse à vos oreilles les cris des femmes éplorées. Vous auriez vu sur l'esplanade du château des mains levées vers le ciel; mais il n'y aurait pas eu un mot de vrai. Le fait est que nous n'éprouvâmes d'autre tempête que celle du premier livre de Virgile, que l'un des élèves de l'abbé nous récita par cœur; et telle fut la fin de notre première sortie ou promenade. (Diderot $3: 138$ )

La grande différence cependant entre les Salons et Jacques le fataliste réside dans le fait que, dans le domaine romanesque, Diderot peut jouir d'une liberté totale d'expression: dans le domaine de la critique d'art. il se voit obligé de maintenir un point de contact avec le tableau qu'il commente. Et la promenade de Vernet est importante précisément parce qu'elle représente en quelque sorte un stade entre les deux genres. C'est effectivement à partir de 1767 que les formes expressives de la critique d'art et le problème de l'expression se transforment en problèmes de l'expression romanesque. 


\section{Conclusion}

En somme, il semble que pour Diderot, le triomphe de la magie picturale, c'est lorsqu' on oublie que l'on se trouve devant un tableau. L'admiration pour le peintre exige que l'on parcoure à loisir un univers, que l'on y discoure à perte de vue, sans oublier de revenir au point de départ.

La technique utilisée par Diderot dans les Salons s'explique donc ainsi : pour rendre compte d'un tableau, il ne faut pas vouloir le restituer " tel qu'il est » - entreprise vaine -, mais au contraire le "réfléchir " aussi subjectivement que possible. C"est ainsi que les tableaux du Louvre se transcrivent par l'imagination de Diderot, dans les représentations qu'il s'en fait. Ses lecteurs n'ignorent pas que, pour lui, rendre compte c'est réinventer, modifier, reconstruire. Ecrire, c'est souvent traduire, annoter dans les marges, reproduire les propos d'autrui ou introduire des digressions qui, à l'occasion, deviennent œuvre. C'est aussi la manière propre à Diderot d'assister à une représentation : il intervient, et, s'il peut, il glisse sur la scène. C'est enfin son mode de lecteur : il écrit à son tour, il récrit. C'est sa façon la plus personnelle de comprendre: il transforme, bref, c'est ainsi qu'il entre dans un texte et qu’il le critique. Il y appose sa marque et, en toute innocence, semble-t-il, se l'approprie. Il se fait même mérite de cette intervention. Non qu'il parasite son objet : il le métamorphose de sa participation active. Avec lui, nous voyons transposée en paroles non pas l'image qu 'il a vue, mais une autre, idéalisée qu'il " compose » lui-même. En se référant à une œuvre connue, Diderot ouvre la voie à la " re-création » du tableau qu'il commente. Mis en présence d'une image de la réalité, il ne s`en contente pas; mais, prenant cette image comme prétexte et point de départ, il imagine et crée un monde qui tienne compte de cette réalité apparente, mais qui satisfasse en même temps des exigences plus personnelles. Cette affabulation à partir d'une représentation visuelle est une des clés de la création romanesque chez Diderot.

Ainsi, Diderot pousse jusqu'à la parodie un procédé narratif plus formel que réel. Il est normal que le narrateur imprime à son récit un ton, un éclairage, une coloration où transparaissent ses propres sentiments : dans une certaine mesure, il juge en racontant. Normal aussi qu'il prenne à témoin celui à qui il se confie, lecteur ou auditeur. Mais ce qui devait être présence légère et discrète se convertit avec Diderot en un véritable envahissement : l'auteur et le lecteur sortent des coulisses et $s$ 'installent sur la scène avec les acteurs.

Mais, après avoir crée l'illusion romanesque, parfois même au moment précis où il la creee, Diderot semble s ingénier à la rompre. Il secoue son lecteur en le faisant constamment passer d'un degré de fiction à un autre. La rupture lui paraît aussi indispensable à l'illusion romanesque qu'elle l'est à la mystification: dans les deux cas, elle joue d'ailleurs le même rôle, qui est de révéler la tromperie en quittant le terrain de l'imaginaire pour revenir à celui du réel. C'est ainsi que peuvent s'expliquer les fausses bévues et les contradictions volontaires, dont le but est de rompre l'illusion, de ramener le lecteur au monde du réel, en un mot, de le démystifier. De là l'importance de toute forme de rupture, qui constitue toujours un détachement par rapport à l'illusion, une reconnaissance de la tromperie comme telle, une prise de conscience démystificatrice.

En somme, meneur de jeu ou démiurge. le mystificateur crée un monde et l'abandonne, à son gré ; il s'en proclame l'auteur et révèle les dessous de sa création, détrompant ainsi celui à qui il s`adresse. C'est donc par le mensonge et la fausseté que 
Zeina Hakim

Diderot prétend accéder à la vérité. De même, il atteint le réel en passant par l'imaginaire: les représentations du monde que nous offrent sa vision subjective nous font déboucher sur une réalité complexe à laquelle la pluralité des points de vue finit par rendre une objectivité toute relative.

\section{Notes}

' C'est le cas, par exemple, dans sa description du Mariage de la V'ierge de Deshays : « Je ne suis pas de l'avis du philosophe sur les sujets que fournit la partie historique de nos livres sacrés. [...] Quant aux sujets historiques tirés de I'Ancien et du Nouveau Testament, j'avoue qu'ils ont de la simplicité, et que les nourrices en peuvent faire d'assez bons contes pour l'amusement des enfants. Je vais blasphémer à l'occasion du tableau du Mariage de la I'ierge. La Vierge de Deshays est bien, très bien, mais si j'osais, je dirais que le bonhomme Joseph a l'air de lui dire: Allons donc, ne fais pas l'enfant ; je dirais que la sainte Anne est si enfoncée qu'elle ne fait pas du tout l'effet de la mère, mais d'un personnage très subalterne. D'ailleurs, mon pasteur, M. Baer, m'a fait remarquer dans ce tableau de terribles bévues contre le costume. Mais il n'y a qu'un pasteur hérétique qui puisse relever de ces fautes-là, et les prêtres catholiques sont trop ignorants pour en être choqués. Mais laissons au bon catholique Diderot poursuivre sa carrière.» (Diderot 1:215).

2 Diderot, dans ce rêve, se trouve dans la caverne de Platon, parmi des hommes enchaînés. A l'exception de quelques individus qui tentent de se libérer. l'auditoire est parfaitement heureux. La projection des images se produit à peu près comme dans Platon: à travers de "petites figures transparentes et colorées ", le spectacle qu'ils projettent, sur la " toile immense " qui tapisse le fond de la caverne, représente " toutes les scènes comiques, tragiques et burlesques de la vie " (Diderot 2: 189)

${ }^{3}$ Diderot s'adresse en effet aux peintres: "Bachelier, mon ami », (Diderot 1:67), " Mon cher monsieur Pierre » (Diderot 1: 113), " Maitre La Grenée » (Diderot 3: 103), tout autant qu'aux personnages représentés sur la toile : "Petite, ouvrez-moi votre cœur, parlez-moi vrai " (Diderot 2: 145).

\section{Bibliographie}

Diderot, Denis. Salons. Ed. Jean Seznec et Jean Adhémar. 4 vols. Oxford : Clarendon Press, 19571967. 
Selected Proceedings from

the UCLA Department of French and

Francophone Studies

Annual Graduate Student Conference

\section{CAMERA OU STYLO: A PROBLEMATIC DIALOGUE?}

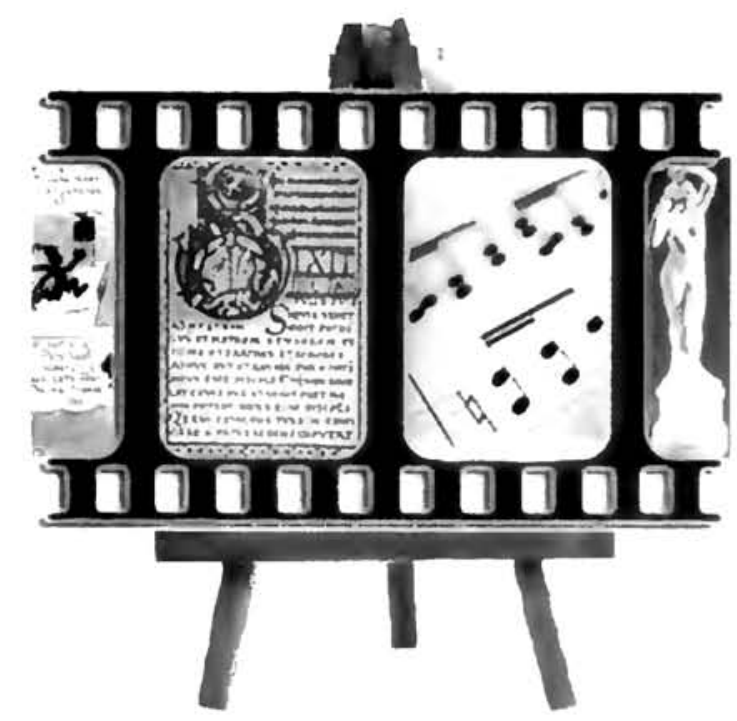

\section{Paroles Gelées \\ UCLA French Studies}

Volume 21

Spring 2004 
Selected Proceedings from

the UCLA Department of French and

Francophone Studies

Annual Graduate Student Conference

\section{CAMERA OU STYLO: A PROBLEMATIC DIALOGUE?}

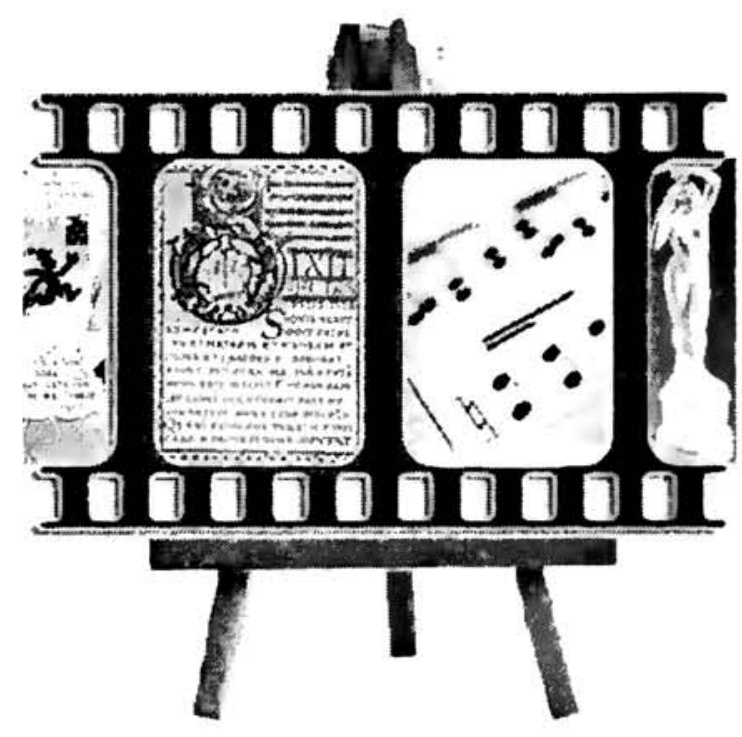

\section{Paroles Gelées UCLA French Studies}

Volume 2I

Spring 2004 
CAMERA OU STYLO: A PROBLEMATIC DIALOGUE?

Selected Proceedings from

the UCLA Department of French and Francophone Studies Eighth Annual Interdisciplinary Graduate Student Conference

October 3-4, 2003

Ce serait le moment de philosopher et de rechercher si, par hasard, se trouvait ici lendroit où de telles paroles dégèlent.

Rabelais, Le Quart Livre.

Paroles Gelées

UCLA French Studies

Volume 21

Spring 2004 
Editor-in-Chief: Vera Klekovkina

Assistant Editor: Nadège Veldwachter

Editorial Board:
Laurence Denié
William T. Hendel
Amy Marczewski
Brice Tabeling
Elizabeth Vitanza

Sponsors: UCLA Graduate Students Association

Albert and Elaine Borchard Foundation

UCLA Campus Programs Committee

UCL $\triangle$ Center for European \& Russian Studies

UCLA Center for Modern \& Contemporary Studies

French Consulate, Los Angeles

UCLA Department of French \& Francophone Studies

Paroles Gelées was established in 1983 by its founding editor, Kathryn Bailey. The journal is managed and edited by the French Graduate Students' Association and published annually under the auspices of the Department of French and Francophone Studies at University of Califormia, Los Angeles. Over the years, Paroles Gelées has concentrated on the publication of selected proceedings from our Annual Graduate Student Conference.

For more information regarding previous issues of Paroles Gelées and our conference, please visit our home page at:

http: www french.ucla.edu gradconf

Or write to us at the following address:

Paroles Gelées

Department of French \& Francophone Studies

212 Royce Hall

Box 951550

Los Angeles, CA 90095-1550

(310) $825-1145$

Subscription price (per issue):

$\$ 12$ for individuals

$\$ 14$ for institutions

$\$ 16$ for international orders

Please make your check payable to: ASUCLA / Paroles Gelées and send it to the aforementioned address.

Cover illustration provided by Vera Klekovkina. Editor-in-Chief.

Copyright $@$ Paroles Gele's 2003-2004 by the Regents of the University of California. ISSN 
Acknowledgements:

Vera Klekovkina, UCLA

- Camera ou Stylo: A Problematic Dialogue? vi-vii

Introduction:

Ben Stoltzfus, UC Riverside

- Introducing Alain Robbe-Grillet 1

Andrea Loselle, UCLA

- Introduction to the Selected Papers 4

Keynote Address:

Alain Robbe-Grillet, de l'Académie française

- Image réelle, image textuelle, image virtuelle 6

Selected Papers:

Anthony Abiragi, New York University

- Mallarmé and the Uprooting of Vision 8

Madalina Akli, Rice University

- Les mots et les couleurs en mouvement $\quad 14$

Mette Gieskes, University of Texas

- Liberating Structures: Non-Visual Systems in the Art of Sol LeWitt 23

Anastasia Graf, Princeton University

- Reading/Writing the Dialectical Image-Walter Benjamin and Osip Mandel'stam

Zeina Hakim, Columbia University

- Rhétorique verbale et visuelle dans les Salons de Diderot

William T. Hendel, UCLA

- The Theatrical Representation of Landscape in Rousseau's La Nouvelle Héloïse

Loli Tsan, UCLA

- Le Jeu du je et du miroir dans la Délie de Maurice Scève

Annex:

Alison Rice, UCLA

- Mémoires du «bled » : Entretien avec Alain Ricard 